\title{
Chapter 35 \\ The Role of Peroxisomes in Plant \\ Reproductive Processes
}

\author{
Shino Goto-Yamada, Shoji Mano, and Mikio Nishimura
}

\begin{abstract}
Peroxisomes are ubiquitous organelles found in eukaryotic cells. These organelles perform important metabolic functions required for normal development. Peroxisomes play crucial roles in flowering plants, functioning in such processes as lipid metabolism, photorespiration, and the production of phytohormones, rendering these organelles indispensable for normal plant growth. In addition to functioning in vegetative tissues, recent studies have shown that peroxisomes play essential roles in reproductive processes. This chapter describes the analysis of peroxisomes in gametophytes, using the approach of organelle visualization in reproductive tissues, which reveals organelle distribution in gametophytes and the dynamics of peroxisomes in pollen cells. Studies using mutants defective in peroxisomal functions demonstrate the importance of peroxisomes to pollen fertility and pollen tube elongation in pistils. We also summarize the relationship between peroxisomal function and reproductive processes in plants. Peroxisomes may contribute to pollen fertility by generating energy for pollen tube growth and producing signaling molecules required for pollen tube elongation and orientation. Moreover, peroxisomes may be involved in male-female gametophyte recognition.
\end{abstract}

Keywords Fluorescent protein $\bullet$ Peroxisome $\bullet$ Plant $\bullet$ Reproduction

\footnotetext{
S. Goto-Yamada

Department of Cell Biology, National Institute for Basic Biology, Okazaki 444-8585, Japan

S. Mano $(\bowtie) \bullet$ M. Nishimura

Department of Cell Biology, National Institute for Basic Biology, Okazaki 444-8585, Japan

Department of Basic Biology, School of Life Science, The Graduate University

for Advanced Studies, Okazaki 444-8585, Japan

e-mail: mano@nibb.ac.jp
} 


\subsection{Introduction}

Peroxisomes are single membrane-bound organelles that are ubiquitous in eukaryotic cells. As peroxisomes contribute to lipid metabolism via $\beta$-oxidation activities in various organisms, including animals and plants, these organelles are essential for survival. Peroxisomes have a variety of functions, such as polyamine metabolism (Nishikawa et al. 2000; Wu et al. 2003; Kamada-Nobusada et al. 2008), the synthesis of various secondary metabolites (Imazaki et al. 2010; Meijer et al. 2010), and vitamin production (Babujee et al. 2010; Tanabe et al. 2011), in addition to lipid metabolism, which demonstrates that peroxisomes are involved in biological processes at various developmental stages. Recent analyses using various mutants showed that defects in peroxisomal function result in the failure of reproductive processes (Footitt et al. 2007; Boisson-Dernier et al. 2008; Wu et al. 2010). This chapter focuses on the relationship between peroxisomal functions and reproductive processes in plants.

\subsection{Peroxisomes in Higher Plants}

\subsubsection{Peroxisomal Functions During Plant Development}

In plants, peroxisomes appear in various tissues as globular organelles of approximately $1-1.5 \mu \mathrm{m}$. Several types of peroxisomes are found in plants, including glyoxysomes and leaf peroxisomes (Kamada et al. 2003). Plant peroxisomes adapt to environmental and developmental changes (Nishimura et al. 1993). Peroxisomes in etiolated cotyledons are referred to as glyoxysomes. These organelles are responsible for the conversion of fatty acids to sucrose, which provides energy for seedling growth after germination (Fig. 35.1a). In photosynthetic leaves, a functional transition from glyoxysomes to leaf peroxisomes takes place (Fig. 35.1b) (Nishimura et al. 1993). Leaf peroxisomes contain enzymes that function in the glycolate pathway and are active during photorespiration. This function is indispensable for scavenging byproducts produced by photosynthesis and is required for normal plant growth (Tolbert 1981). Moreover, plant peroxisomes contribute to the generation and detoxification of reactive oxygen species (ROS), the biosynthesis of plant hormones, such as jasmonate and auxin, and the metabolism of polyamines (Kamada-Nobusada et al. 2008). Studies of various Arabidopsis mutants with defective peroxisomes have demonstrated that peroxisomal functions and biogenesis are essential for plant growth (Hayashi et al. 1998, 2002; Mano et al. 2004, 2006; Goto et al. 2011).

Because all peroxisomal proteins are encoded by the nuclear genome, peroxisomal proteins are translated on free ribosomes in the cytosol. These proteins are then transported to peroxisomes when their peroxisomal targeting signals 1 or 2 (PTS1 or PTS2) are recognized by the cytosolic receptors PEROXIN 5 (PEX5) and PEX7, respectively, which direct the proteins to the peroxisomal membrane. Constitutive expression of the gene encoding the PTS1 fused green fluorescent protein (GFP) construct under the control of the CaMV 35S promoter results in the 

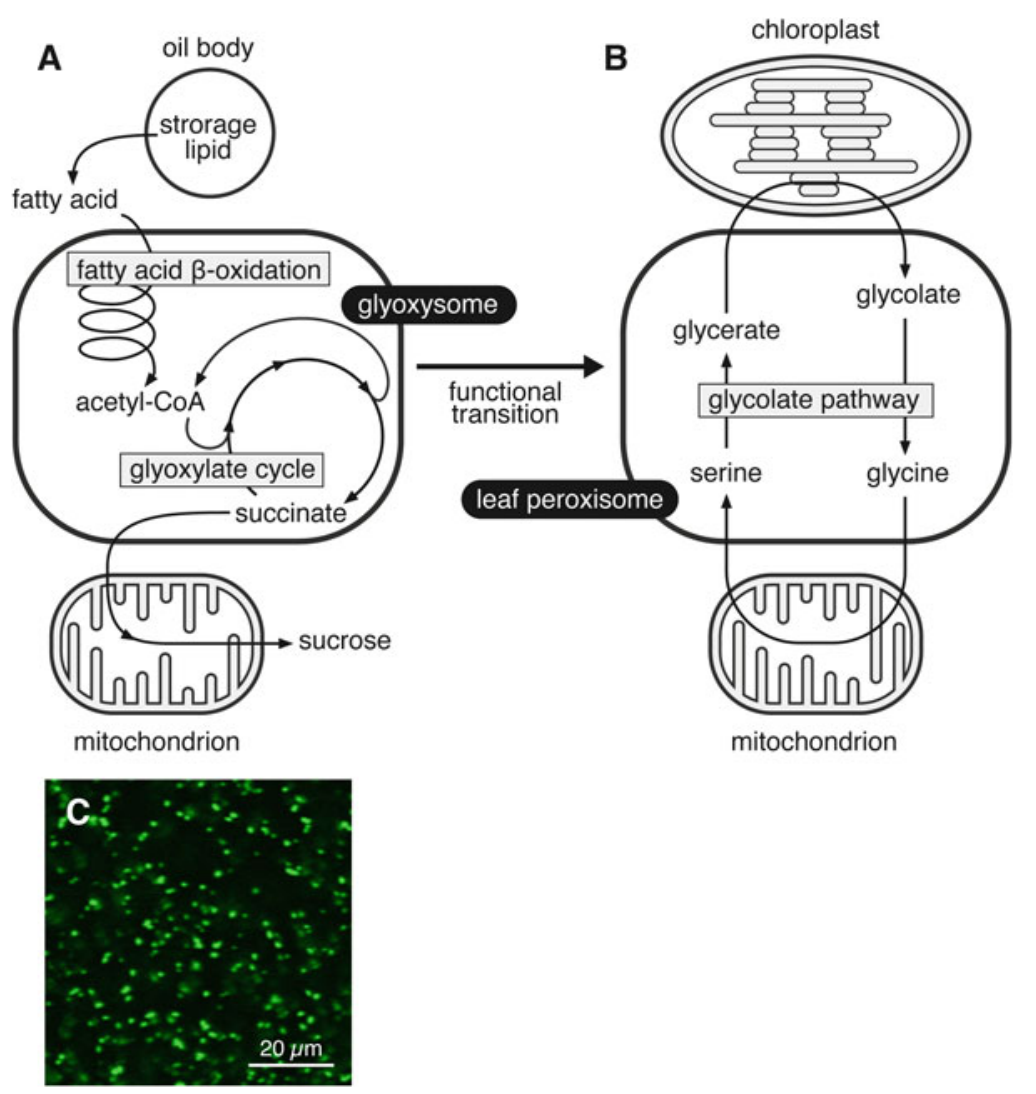

Fig. 35.1 Plant peroxisomes. (a, b) Metabolic pathways in peroxisomes. Lipid metabolism in glyoxysomes (a) and photorespiration in leaf peroxisomes (b). After the plant receives light, a functional transition from glyoxysomes to leaf peroxisomes occurs. (c) GFP-labeled peroxisomes in leaves of transgenic Arabidopsis expressing GFP-PTS1 under the control of the CaMV 35S promoter

transport of this protein to the peroxisomes; GFP in the peroxisomes is detectable in almost all cells when visualized under a fluorescence microscope (Fig. 35.1c) (Mano et al. 2002). This visualization system is useful for a variety of experiments, including the observation of peroxisome dynamics, such as morphology and movement, and the isolation of mutants (Mano et al. 2004, 2006, 2011; Goto et al. 2011).

\subsubsection{Peroxisomes in Plant Reproductive Tissues}

Electron microscopic observation has revealed that peroxisomes exist in both vegetative and reproductive tissues. Many vesicles thought to be oil bodies are present in pollen grains, and peroxisomes are found among them (Fig. 35.2a, e). Pollen peroxisomes appear as spherical structures, and they are smaller than peroxisomes in vegetative tissues, with an average size of approximately $500 \mathrm{~nm}$. To understand the 

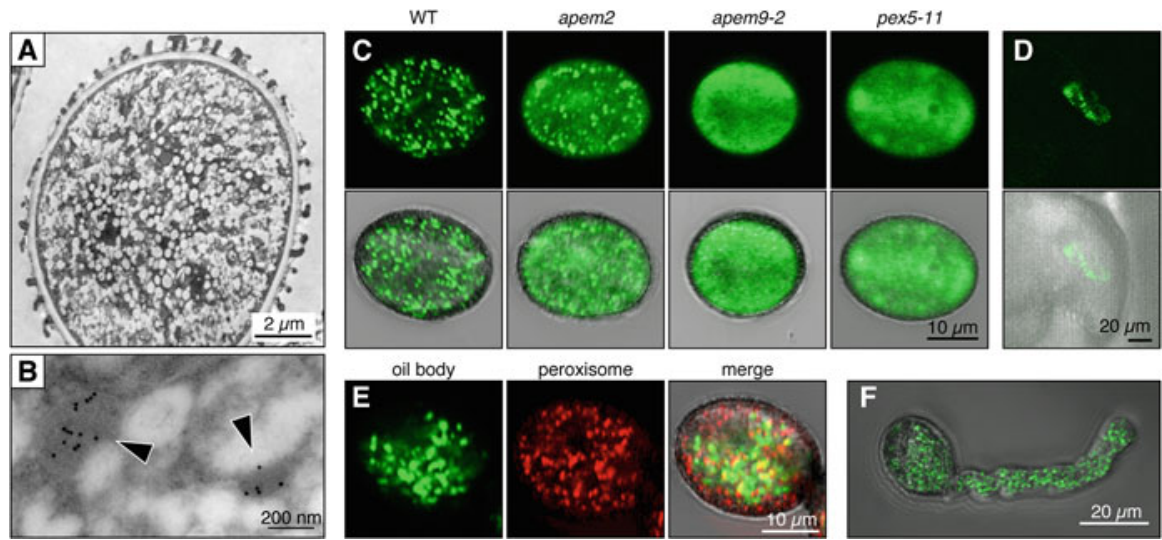

peroxisome

merge
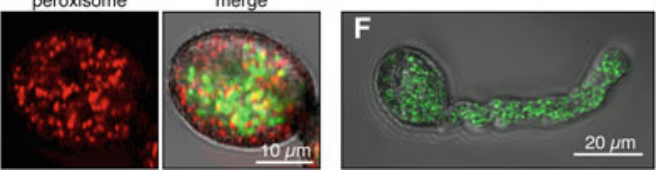

Fig. 35.2 Peroxisomes in reproductive tissues. (a) Electron micrograph of Arabidopsis pollen. (b) Immunoelectron micrograph of pollen from transgenic Arabidopsis harboring Lat52p:GFPPTS1. Arrowheads indicate peroxisomes, which were immunogold labeled with anti-GFP antibodies. (c) GFP fluorescence pattern in wild-type and peroxisome-defective mutants apem2, pex5-11, and apem9-2, which express GFP-PTS1. The pex5-11 and apem9-2 represent the T-DNA insertion lines SALK_018579 and SALK-132193, respectively. Both mutants are unable to produce homozygotes, and heterozygotes produce two types of pollen that exhibit different phenotypes, including wild-type-like spherical structures and mutant structures exhibiting cytosolic fluorescence; only pollen grains of the mutant are shown in this figure. (d) Peroxisomes in egg cell of female gametophyte. Peroxisomes were visualized using DD45p:GFP-PTS1, with the expression of GFPPTS1 under the control of the DD45 promoter. (e) Localization of oil bodies and peroxisomes in pollen. Oil bodies were visualized with GFP-labeled oleosin, a membrane protein present in oil bodies. Peroxisomes were visualized with TagRFP-PTS1. (f) Subcellular localization of peroxisomes in elongating pollen tube

temporal and spatial behavior of peroxisomes during reproductive processes, we generated transgenic Arabidopsis-expressing peroxisome-targeted fluorescent protein in pollen and egg cells using the Lat52 and DD45 (Twell et al. 1991; Steffen et al. 2007) promoters, respectively. Because the CaMV 35S promoter does not function properly in these cells, Lat52 and DD45 promoter-controlled GFP-PTS1 (Lat52p:GFP-PTS1 or DD45p:GFP-PTS1) was used to visualize peroxisomes in pollen and egg cell, respectively. Using these constructs, we could easily observe peroxisomal movement and distribution during the reproductive processes (Fig 35.2c,d). In mature pollen grains, the peroxisomes are quite immobile, but after pollen germination, the peroxisomes move in the direction of pollen tube growth (Fig. 35.2f). The peroxisomes then move through the pollen tube that reaches the female gametophyte.

\subsection{Key Genes of Peroxisome Biogenesis Also Function in Pollen Cells}

In flowering plants and in animals, peroxisomal functions are essential for normal development and survival. In humans, peroxisome biogenesis disorders, including Zellwerger syndrome, are fatal genetic disorders (Fujiki 2000). In plants, defects of 
peroxisomal biogenesis and function result in various defects, including the failure to germinate without sucrose (Hayashi et al. 1998; Hayashi et al. 2002), dwarfism (Mano et al. 2004, 2006; Goto et al. 2011), and embryonic lethality (Schumann et al. 2003; Sparkes et al. 2003; Fan et al. 2005). Moreover, recent studies have revealed that defects in peroxisomes result in a decrease of pollen fertility and failures in gametophyte recognition (Boisson-Dernier et al. 2008), indicating that explication of peroxisomal functions is important for understanding reproductive processes in plants.

As reported previously, we isolated a number of Arabidopsis apem (aberrant peroxisome morphology) mutants expressing 35Sp:GFP-PTS1 that had different patterns of GFP fluorescence from the parental GFP-PTS1 plants (Mano et al. 2004, 2006, 2011; Goto et al. 2011). To understand the role of peroxisomes in pollen fertility, we expressed the Lat52p:GFP-PTS1 transgene in various mutants defective in peroxisomal biogenesis and function, including the apem mutants. Of these mutants, both apem 2 and apem 9 have mutations in the PEX genes, which are key genes of peroxisome biogenesis. APEM2 and APEM 9 encode PEX13 and plant-specific factor, respectively, and both proteins are involved in peroxisomal protein transport. Therefore, in both mutants, GFP-PTS1 protein is accumulated in the cytosol as well as in peroxisomes (Mano et al. 2006; Goto et al. 2011). As shown in Fig. 35.2c, the pattern of GFP fluorescence in the pollen cells of both mutants is similar to that in vegetative tissues; GFP fluorescence is observed in the cytosol, whereas GFP is observed only in peroxisomes in the wild-type background. A similar pattern is observed in the pollen of a mutant defective in PEX5, which encodes a receptor of peroxisomal matrix protein and is essential for peroxisomal protein transport. As shown in Fig. 35.2c, GFP-PTS1 protein is accumulated in the cytosol in pollen cells of the pex5-11 mutant. These results show that peroxisomal protein transport functions in pollen peroxisomes and that peroxisome biogenesis factors, such as PEXs, act in pollen cells.

\subsection{Effects of Peroxisome Defects on Male Gametes}

To examine the contribution of peroxisomal function to reproductive process, the peroxisome-defective apem 9 mutant was employed. First, to investigate the fertility of gametes in peroxisome-defective mutants, reciprocal crosses of apem $9-2 /+$ with wild-type Columbia (Col) were performed. When apem $9-2 /+$ pollen was used to pollinate Col pistils, only $14.6 \%$ [transmission efficiency (TE) $=17.1$, $n=96]$ of the seeds had the apem $9-2 /+$ genotype, whereas when apem $9-2 /+$ pistils were pollinated with Col pollen, $45.5 \%(\mathrm{TE}=83.3, n=44)$ of the seeds had the apem $9-2 /+$ genotype (Table 35.1). These results show that the transmission of the mutation through the female gametophyte is not impaired, and apem 9 ovules are fertile. However, pollen development and function are severely affected in the apem 9 mutant. 
Table 35.1 Reciprocal crosses between peroxisome mutants and wild-type plants

\begin{tabular}{lllll}
\hline Mutant name & Gene & TE female $(\%)^{\mathrm{a}}$ & TE male $(\%)^{\mathrm{a}}$ & Reference \\
\hline pex5_11 $^{\mathrm{b}}$ & PEROXIN5 & 110.0 & 30.2 & This study \\
apem9-2 $^{\mathrm{c}}$ & APEM9 & 83.3 & 17.1 & This study \\
amclapem2 $^{\text {a }}$ & PEROXIN13 & 82.8 & 45.3 & Boisson-Dernier et al. (2008) \\
${\text { cts }-1^{\mathrm{d}}}_{\text {cts }^{\mathrm{d}}}^{\mathrm{d}}$ & COMATOSE & 93.9 & 21.6 & Footitt et al. (2007) \\
\hline T & COMATOSE & 111.3 & 10.3 & Footitt et al. (2007) \\
\hline
\end{tabular}

${ }^{a}$ Transmission efficiencies (TE) were calculated according to Howden et al. (1998)

bpex5_11 represents SALK_018579

capem 9 -2 represents SALK-132193

${ }^{\mathrm{d}}$ cts- 1 and $c t s-2$ are allelic to ped $3 /$ pxal, which is defective in peroxisomal $\mathrm{ABC}$ transporter
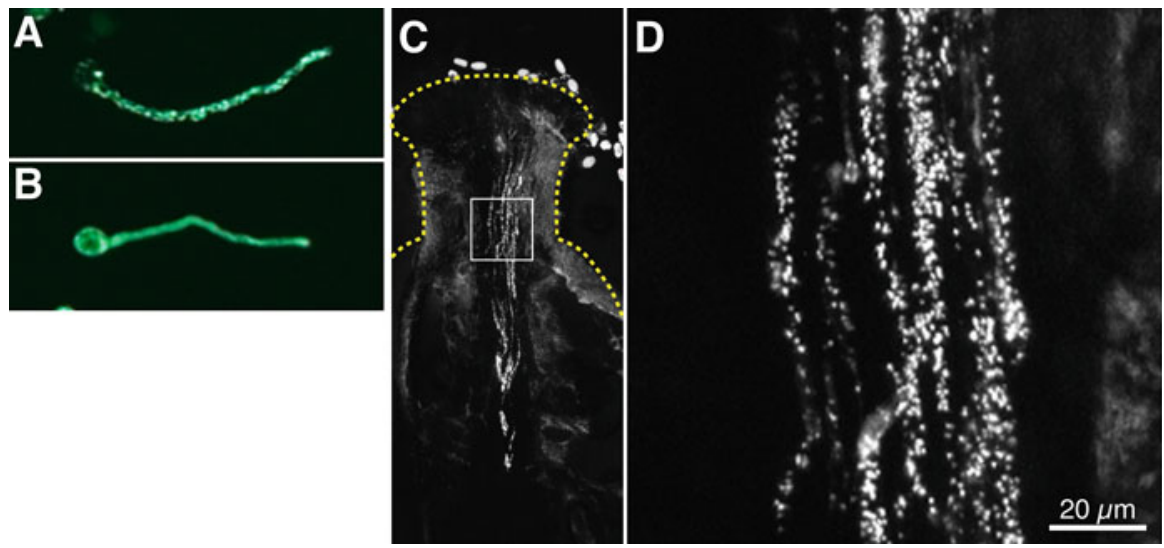

Fig. 35.3 Analysis of pollen tube elongation in apem 9-2 mutant and wild-type plant. (a, b) Fluorescence images of elongating wild-type (a) and apem9-2 (b) pollen tubes grown in vitro. (c) In vivo pollen germination assay. Mutant apem $9-2 /+$ pollens were used to pollinate a wild-type pistil, and GFP fluorescence was observed with a laser-scanning confocal microscope $1 \mathrm{~h}$ after pollination. (d) Magnified image of the area enclosed by the white box in $\mathbf{c}$

Pollen fertilization occurs via the following steps: (1) pollen maturation, (2) pollen germination and elongation of the pollen tube, (3) guidance of the pollen tube to the ovule, and (4) fertilization with the ovule. To determine whether the reduced male transmission of the apem 9-2 mutation was caused by a pollen germination defect, and to identify which step(s) in fertilization are disturbed in apem $9-2$ pollen, an in vitro pollen germination assay was performed, and the numbers of germinated wild-type (+) and apem $9-2$ pollen were counted. There was no obvious difference in the number of germinated pollen grains between wild-type and apem9-2 pollen, indicating that apem9-2 pollen matured successfully, as did the wild-type pollen, and had the ability to germinate under artificial germination conditions (Fig. 35.3a,b). However, in the pollen germination assay in vivo, in which pollen was germinated on wild-type stigmas, the apem9-2 pollen tubes, unlike the wild-type pollen tubes, failed to elongate in the top parts of the pistils within $1 \mathrm{~h}$ of pollination (Fig. 35.3c,d). Based on these results, we determined that the defect in 
the APEM9 gene may decrease the efficiency of pollen fertility caused by a delay in pollen tube elongation in vivo or a failure of pollen tube penetration into the papilla cells, indicating that peroxisome biogenesis and function are required for pollen fertility.

\subsection{Relationship Between Peroxisomal Function and Reproduction}

In addition to the apem 9 mutant, other peroxisome mutants also show defects in pollen fertility. Table 35.1 shows the results of reciprocal crosses between peroxisome mutants. Although the female TEs are almost normal in these mutants, the male TEs are obviously decreased. These results indicate that peroxisomal functions are required for pollen fertility. This section reviews the relationship between peroxisomal functions and reproductive processes, including pollen fertility.

\subsubsection{Peroxisomal $\beta$-Oxidation and Pollen Fertility}

Fatty acid $\beta$-oxidation is one of the major metabolic processes that occur in peroxisomes. Fatty acid $\beta$-oxidation is required for the metabolism of lipids, as well as the conversion of fatty acid derivates into phytohormones, such as auxin and jasmonic acid (JA) (Wain and Wightman 1954; Sanders et al. 2000; Stintzi and Browse 2000). JA plays a crucial role as a signaling molecule during plant growth and development and in plant responses to biotic and abiotic stress. Moreover, JA contributes to reproductive processes. Peroxisome-targeted OPR3 (12-oxophytodienoic acid reductase 3) is one of the enzymes required for JA biosynthesis (Sanders et al. 2000; Stintzi and Browse 2000). The Arabidopsis opr3 mutant shows the male-sterility phenotype, resulting from defects of sufficient elongation of the anther filament, normal anther dehiscence, and the production of viable pollen (Sanders et al. 2000; Stintzi and Browse 2000). These results indicate the importance of peroxisomal $\beta$-oxidation in plant reproductive processes.

Another important aspect of fatty acid $\beta$-oxidation is lipid metabolism in seeds. Oilseed plants, such as Arabidopsis and pumpkin, convert seed-storage lipids to sucrose during postgerminative growth as energy sources before the plant begins photosynthesis (Fig. 35.1a). Triacylglycerols are accumulated as storage lipids in organelles known as oil bodies, which are surrounded by oleosin in seeds. Similarly, numerous oil bodies and peroxisomes are observed in pollen grains when examined by electron (Fig. 35.2a) and fluorescence microscopy (Fig. 35.2e). The similarity between seeds and pollen suggests that fatty acid $\beta$-oxidation provides energy during pollen germination and/or pollen tube elongation. Indeed, the lack of CTS (COMATOSE), an ABC transporter that contributes to the uptake of fatty acids into peroxisomes, results in the suppression of pollen tube elongation (Footitt et al. 2007), 
whereas this mutant pollen tube can elongate in artificial pollen germination medium containing sucrose. The phenotype for suppression of pollen tube elongation is also seen in other mutants defective in fatty acid $\beta$-oxidation, such as KAT2 (3-keto acylCoA thiolase), LACS6, and LACS7 (long-chain acyl-CoA synthetase 6 and 7), which demonstrates the importance of peroxisomal fatty acid $\beta$-oxidation in pollen fertility and pollen tube elongation (Footitt et al. 2007).

\subsubsection{Peroxisome Biogenesis and Male-Female Gametophyte Recognition}

A recent study has revealed that peroxisomes are involved in male-female gametophyte recognition (Boisson-Dernier et al. 2008). The amc (abstinence by mutual consent) mutant was originally identified as a mutant with defective in male-female gamete recognition. In addition to decreased pollen fertility, the amc mutant exhibits impaired fertilization only when both male and female gametophytes have mutations in $A M C$. In wild-type plants, pollen germinates after it attaches to a pistil. The pollen tube then elongates, reaches one of the two synergid cells of the female gametophyte, and ruptures to release two sperm cells. However, the amc mutant shows impaired pollen tube reception when an amc pollen tube reaches an $a m c$ female gametophyte, resulting in the continued growth of the pollen tube in the synergid cell. As the pollen tube fails to rupture, the sperm cells are not released, and homozygous mutants never develop. Interestingly, the $A M C$ gene is allelic to apem2 (Mano et al. 2006). Therefore, the amc mutant is defective in one of the peroxisome biogenesis factors, PEX13, which is required for peroxisomal protein transport. The $A M C / P E X 13$ gene is transiently expressed in both male and female gametophytes during fertilization (Boisson-Dernier et al. 2008). As PEX13 is one of the peroxisome biogenesis factors, these results indicate that peroxisome biogenesis plays an unexpected key role in gametophyte recognition.

\subsubsection{Peroxisome-Derived ROS and NO and Reproduction}

ROS and nitric oxide (NO) are signaling molecules; peroxisomes are involved in the production of these compounds. A recent study has revealed that peroxisometargeted PAO (polyamine oxidase) contributes to pollen tube elongation in association with ROS production (Wu et al. 2010). This study illustrates that PAO regulates pollen tube elongation during the oxidation of spermidine, one of the polyamines, and generates hydrogen peroxide $\left(\mathrm{H}_{2} \mathrm{O}_{2}\right)$, which triggers the opening of the hyperpolarization-activated $\mathrm{Ca}^{2+}$-permeable channels in pollen. The Arabidopsis genome has five isoforms of PAO, and three of these (PAO2, PAO3, and PAO4) are localized to peroxisomes (Kamada-Nobusada et al. 2008). The pao3 mutant exhibits 
suppressed pollen tube elongation and decreased seed numbers, indicating the importance of peroxisome function, combined with ROS production, in plant reproduction. The details of how PAO-mediated $\mathrm{H}_{2} \mathrm{O}_{2}$ production contributes to pollen fertility will become clearer in future studies. In addition, Prado et al. reported that NO is generated in peroxisomes in the pollen tube, and NO regulates pollen tube orientation in vitro (Prado et al. 2004). Although the activity of NOS (nitrogen oxide synthase) is detected in peroxisomes isolated from pea (Pisum sativum) (Barroso et al. 1999), peroxisome-targeted NOS has not been identified yet. The metabolic process leading to NO production is still largely unclear and should be investigated in the future.

\subsection{Perspectives}

This chapter describes how plant peroxisomes contribute to pollen development, pollen tube elongation, and male-female gametophyte recognition. Although some details about how peroxisomes function during reproduction remain to be elucidated, the importance of peroxisomes in reproductive processes has become clear through the analysis of peroxisome mutants. Defects in peroxisomal biogenesis and metabolism impair sperm development and fertility in Drosophila and other animals as well as in plants (Huyghe et al. 2006; Nenicu et al. 2007; Chen et al. 2010; Nakayama et al. 2011), suggesting that peroxisomes may function in the reproductive processes of various organisms in a similar manner.

In mammalian cells, the $\beta$-oxidation is localized in both peroxisomes and mitochondria. Long- and medium-chain fatty acids were metabolized in peroxisomes, whereas short-chain fatty acids were metabolized in mitochondria but not in peroxisomes. In plants, however, the activity of $\beta$-oxidation is present only in peroxisomes. Therefore, we have to consider the difference along with the similarity of peroxisomal functions between plants and animals. Transdisciplinary investigation that concerns both plant and mammalian peroxisomes will clarify the similarities or differences of peroxisomal functions in the reproductive process among various organisms.

Acknowledgments We thank Dr. Tomoko Igawa in Chiba University for providing the DD45 clone, and Dr. Sheila McCormick in University of California, Berkeley, for providing the Lat52 clone. This work was supported by MEXT KAKENHI of Grant-in-aid for Scientific Research on Innovative Areas, "Elucidating Common Mechanisms of Allogenic Authentication" (No. 22112523), to S.M., and the Japan Society for the Promotion of Science for a Research Fellowship for Young Scientists (No. 22.9) to S.G.-Y. We thank Chinami Yamaguchi, Azusa Matsuda, Masami Araki, Chihiro Nakamori, Maki Kondo, and Dr. Makoto Hayashi from the Division of Cell Mechanisms at the National Institute for Basic Biology for supporting this work, and staff from the Model Plant Research Facility at the National Institute for Basic Biology for plant care.

Open Access: This article is distributed under the terms of the Creative Commons Attribution Noncommercial License which permits any noncommercial use, distribution, and reproduction in any medium, provided the original author(s) and source are credited. 


\section{References}

Babujee L, Wurtz V, Ma C, Lueder F, Soni P, van Dorsselaer A, Reumann S (2010) The proteome map of spinach leaf peroxisomes indicates partial compartmentalization of phylloquinone (vitamin $\mathrm{K}_{1}$ ) biosynthesis in plant peroxisomes. J Exp Bot 61(5):1441-1453

Barroso JB, Corpas FJ, Carreras A, Sandalio LM, Valderrama R, Palma JM, Lupianez JA, del Rio LA (1999) Localization of nitric-oxide synthase in plant peroxisomes. J Biol Chem 274(51): 36729-36733

Boisson-Dernier A, Frietsch S, Kim T-H, Dizon MB, Schroeder JI (2008) The peroxin loss-offunction mutation abstinence by mutual consent disrupts male-female gametophyte recognition. Curr Biol 18(1):63-68

Chen H, Liu Z, Huang X (2010) Drosophila models of peroxisomal biogenesis disorder: peroxins are required for spermatogenesis and very-long-chain fatty acid metabolism. Hum Mol Genet 19(3):494-505

Fan J, Quan S, Orth T, Awai C, Chory J, Hu J (2005) The Arabidopsis PEX12 gene is required for peroxisome biogenesis and is essential for development. Plant Physiol 139(1):231-239

Footitt S, Dietrich D, Fait A, Fernie AR, Holdsworth MJ, Baker A, Theodoulou FL (2007) The COMATOSE ATP-binding cassette transporter is required for full fertility in Arabidopsis. Plant Physiol 144(3):1467-1480

Fujiki Y (2000) Peroxisome biogenesis and peroxisome biogenesis disorders. FEBS Lett 476(1-2):42-46

Goto S, Mano S, Nakamori C, Nishimura M (2011) Arabidopsis ABERRANT PEROXISOME MORPHOLOGY9 is a peroxin that recruits the PEX1-PEX6 complex to peroxisomes. Plant Cell 23(4):1573-1587

Hayashi M, Toriyama K, Kondo M, Nishimura M (1998) 2,4-Dichlorophenoxybutyric acidresistant mutants of Arabidopsis have defects in glyoxysomal fatty acid $\beta$-oxidation. Plant Cell 10(2): 183-195

Hayashi M, Nito K, Takei-Hoshi R, Yagi M, Kondo M, Suenaga A, Yamaya T, Nishimura M (2002) Ped3p is a peroxisomal ATP-binding cassette transporter that might supply substrates for fatty acid $\beta$-oxidation. Plant Cell Physiol 43(1):1-11

Howden R, Park SK, Moore JM, Orme J, Grossniklaus U, Twell D (1998) Selection of T-DNAtagged male and female gametophytic mutants by segregation distortion in Arabidopsis. Genetics 149(2):621-631

Huyghe S, Schmalbruch H, De Gendt K, Verhoeven G, Guillou F, Van Veldhoven PP, Baes M (2006) Peroxisomal multifunctional protein 2 is essential for lipid homeostasis in sertoli cells and male fertility in mice. Endocrinology 147(5):2228-2236

Imazaki A, Tanaka A, Harimoto Y, Yamamoto M, Akimitsu K, Park P, Tsuge T (2010) Contribution of peroxisomes to secondary metabolism and pathogenicity in the fungal plant pathogen Alternaria alternata. Eukaryot Cell 9(5):682-694

Kamada T, Nito K, Hayashi H, Mano S, Hayashi M, Nishimura M (2003) Functional differentiation of peroxisomes revealed by expression profiles of peroxisomal genes in Arabidopsis thaliana. Plant Cell Physiol 44(12):1275-1289

Kamada-Nobusada T, Hayashi M, Fukazawa M, Sakakibara H, Nishimura M (2008) A putative peroxisomal polyamine oxidase, AtPAO4, is involved in polyamine catabolism in Arabidopsis thaliana. Plant Cell Physiol 49(9):1272-1282

Mano S, Nakamori C, Hayashi M, Kato A, Kondo M, Nishimura M (2002) Distribution and characterization of peroxisomes in Arabidopsis by visualization with GFP: dynamic morphology and actin-dependent movement. Plant Cell Physiol 43(3):331-341

Mano S, Nakamori C, Kondo M, Hayashi M, Nishimura M (2004) An Arabidopsis dynaminrelated protein, DRP3A, controls both peroxisomal and mitochondrial division. Plant J 38(3): 487-498

Mano S, Nakamori C, Nito K, Kondo M, Nishimura M (2006) The Arabidopsis pex12 and pex13 mutants are defective in both PTS1- and PTS2-dependent protein transport to peroxisomes. Plant J 47(4):604-618 
Mano S, Nakamori C, Fukao Y, Araki M, Matsuda A, Kondo M, Nishimura M (2011) A defect of peroxisomal membrane protein 38 causes enlargement of peroxisomes. Plant Cell Physiol 52(12):2157-2172

Meijer WH, Gidijala L, Fekken S, Kiel JA, van den Berg MA, Lascaris R, Bovenberg RA, van der Klei IJ (2010) Peroxisomes are required for efficient penicillin biosynthesis in Penicillium chrysogenum. Appl Environ Microbiol 76(17):5702-5709

Nakayama M, Sato H, Okuda T, Fujisawa N, Kono N, Arai H, Suzuki E, Umeda M, Ishikawa HO, Matsuno K (2011) Drosophila carrying pex3 or pex 16 mutations are models of Zellweger syndrome that reflect its symptoms associated with the absence of peroxisomes. PLoS One 6(8):e22984

Nenicu A, Luers GH, Kovacs W, David M, Zimmer A, Bergmann M, Baumgart-Vogt E (2007) Peroxisomes in human and mouse testis: differential expression of peroxisomal proteins in germ cells and distinct somatic cell types of the testis. Biol Reprod 77(6):1060-1072

Nishikawa M, Hagishita T, Yurimoto H, Kato N, Sakai Y, Hatanaka T (2000) Primary structure and expression of peroxisomal acetylspermidine oxidase in the methylotrophic yeast Candida boidinii. FEBS Lett 476(3):150-154

Nishimura M, Takeuchi Y, De Bellis L, Hara-Nishimura I (1993) Leaf peroxisomes are directly transformed to glyoxysomes during senescence of pumpkin cotyledons. Protoplasma 175(3-4):131-137

Prado AM, Porterfield DM, Feijo JA (2004) Nitric oxide is involved in growth regulation and reorientation of pollen tubes. Development (Camb) 131(11):2707-2714

Sanders PM, Lee PY, Biesgen C, Boone JD, Beals TP, Weiler EW, Goldberg RB (2000) The Arabidopsis DELAYED DEHISCENCE1 gene encodes an enzyme in the jasmonic acid synthesis pathway. Plant Cell 12(7):1041-1061

Schumann U, Wanner G, Veenhuis M, Schmid M, Gietl C (2003) AthPEX10, ariuclear gene essential for peroxisome and storage organelle formation during Arabidopsis embryogenesis. Proc Natl Acad Sci USA 100(16):9626-9631

Sparkes IA, Brandizzi F, Slocombe SP, El-Shami M, Hawes C, Baker A (2003) An Arabidopsis pex10 null mutant is embryo lethal, implicating peroxisomes in an essential role during plant embryogenesis. Plant Physiol 133(4):1809-1819

Steffen JG, Kang IH, Macfarlane J, Drews GN (2007) Identification of genes expressed in the Arabidopsis female gametophyte. Plant J 51(2):281-292

Stintzi A, Browse J (2000) The Arabidopsis male-sterile mutant, opr3, lacks the 12-oxophytodienoic acid reductase required for jasmonate synthesis. Proc Natl Acad Sci USA 97(19):10625-10630

Tanabe Y, Maruyama J, Yamaoka S, Yahagi D, Matsuo I, Tsutsumi N, Kitamoto K (2011) Peroxisomes are involved in biotin biosynthesis in Aspergillus and Arabidopsis. J Biol Chem 286(35):30455-30461

Tolbert NE (1981) Metabolic pathways in peroxisomes and glyoxysomes. Annu Rev Biochem 50:133-157

Twell D, Yamaguchi J, Wing RA, Ushiba J, McCormick S (1991) Promoter analysis of genes that are coordinately expressed during pollen development reveals pollen-specific enhancer sequences and shared regulatory elements. Gene Dev 5(3):496-507

Wain RL, Wightman F (1954) The growth regulating activity of certain $\omega$-substituted alkyl carboxylic acids in relation to their $\beta$-oxidation within the plant. Proc R Soc Lond B Biol Sci 142(909):525-536

Wu T, Yankovskaya V, McIntire WS (2003) Cloning, sequencing, and heterologous expression of the murine peroxisomal flavoprotein, $N^{1}$-acetylated polyamine oxidase. J Biol Chem 278(23): 20514-20525

Wu J, Shang Z, Jiang X, Moschou PN, Sun W, Roubelakis-Angelakis KA, Zhang S (2010) Spermidine oxidase-derived $\mathrm{H}_{2} \mathrm{O}_{2}$ regulates pollen plasma membrane hyperpolarizationactivated $\mathrm{Ca}^{2+}$-permeable channels and pollen tube growth. Plant J 63(6):1042-1053 